\title{
Philosophiques
}

\section{RICHARD BETT, Pyrrho. His Antecedents and his Legacy, Oxford, Oxford University Press, 2000, 264 p.}

\section{Jean Laberge}

Volume 31, numéro 2, automne 2004

URI : https://id.erudit.org/iderudit/009826ar

DOI : https://doi.org/10.7202/009826ar

Aller au sommaire du numéro

Éditeur(s)

Société de philosophie du Québec

ISSN

0316-2923 (imprimé)

1492-1391 (numérique)

Découvrir la revue

Citer ce compte rendu

Laberge, J. (2004). Compte rendu de [RICHARD BETT, Pyrrho. His Antecedents and his Legacy, Oxford, Oxford University Press, 2000, 264 p.] Philosophiques, 31(2), 452-457. https://doi.org/10.7202/009826ar d'utilisation que vous pouvez consulter en ligne.

https://apropos.erudit.org/fr/usagers/politique-dutilisation/ 
pour Plotin, l'Un n'est pas «l'indéfini tracé en creux par les formes finies, mais le positivement infini; non pas l'en deçà ou l'en-creux du quelque chose, mais l'au-delà positif et insaisissable de toutes choses [...] en amont de la superstructure de l'être et de toute superstructure concevable» (p. 165).

J.-M. Narbonne en conclut que la métaphysique de Plotin est bien, d'un certain point de vue, "une métaphysique de la fondation et par là une onto-théologie ", mais que celle-ci «institue à son sommet un existant tout à fait singulier dont la nature transcende la figure classique de l'étant fondateur». La nouveauté de Plotin n'est donc pas d'avoir interrompu le projet classique d'une recherche du fondement, mais bien de lui avoir communiqué "une dimension nouvelle, celle d'une réflexion arcboutée sur l'infini» (p. 165-166).

JOACHIM LACROSSE

Université libre de Bruxelles et École pratique des hautes études

\section{RICHARD BETT, Pyrrho. His Antecedents and his Legacy, Oxford, Oxford University Press, 2000, 264 p.}

«Tant d'écoles philosophiques disparaissent sans héritier. Il ne reste plus aucun représentant des Académiciens, des anciens comme des plus récents.

Et qui se chargera de transmettre l'héritage de Pyrrhon ?»

Sénèque, Questions naturelles, VII, 32.

Le scepticisme n'est pas une philosophie sortie tout droit pour ainsi dire de pied en cap de l'esprit de Sextus Empiricus, ce philosophe-médecin grec du deuxième siècle de notre ère dont on ne sait que bien peu de choses. Bien sûr, avant lui, il y a Pyrrhon d'Élis, le fondateur du scepticisme, qui vécu entre 365 et 270 avant notre ère. De lui aussi on ne sait à peu près rien, d'autant plus que, comme Socrate, il n'a laissé aucun écrit. Mais si quelqu'un fut sceptique, c'est bien lui, Pyrrhon, l'initiateur du scepticisme — ou plutôt du "pyrrhonisme» comme on désignait ce courant philosophique ancien jusqu'au seizième et dix-septième siècles. Richard Bett entend corriger la tradition sur ce point, car, pour lui, Pyrrhon ne fonde pas son scepticisme sur les limites inhérentes de la connaissance humaine, mais sur la nature intrinsèque de la réalité. En d'autres termes, Pyrrhon serait un métaphysicien qui déclare que la réalité est indéterminée de sorte que, pour cette raison, nos jugements sur les choses ne sont ni vrais ni faux. En somme, et au grand scandale de son lointain héritier, Sextus Empiricus, Pyrrhon serait un vilain "dogmatiste». Bett ne propose donc rien de moins qu'une nouvelle compréhension de l'histoire du scepticisme ancien. L'interprétation qu'il propose s'oppose à l'interprétation conventionnelle qui voit en Pyrrhon le fondateur de la doctrine sceptique, doctrine que ses successeurs, Énésidème et Sextus Empiricus, vont par la suite élaborer sans rien changer quant au fond.

En 1562, le français Henri Estienne - Stéphanus, de son nom latinisé, le même qui édita les œuvres de Platon — publia en latin les Purrhoneioi Hupotuposeis - les Esquisses pyrhoniennes - (dorénavant abrégé par PH d'après le titre original grec) de Sextus Empiricus. L'ouvrage fit alors sensation et exerça par la suite une influence décisive sur le développement de la philosophie européenne. Sextus Empiricus donne 
dans les PH la forme canonique du scepticisme tel que le connaîtra l'Europe à la Renaissance. Le renouveau actuel d'intérêt pour le scepticisme ancien est manifeste comme en témoigne la parution, il y a quelques années à peine (en 1997), de l'édition française critique des PH de Sextus aux Éditions du Seuil, dans une version bilingue grec-français, due aux soins de Pierre Pellegrin qui fait précéder sa traduction d'une copieuse et judicieuse introduction.

On a pris l'habitude de comprendre Pyrrhon au travers de la lunette tardive des PH de Sextus. Or, si Bett a raison, le scepticisme de Pyrrhon diffère de celui d'Énésidème de Cnossos, lequel diffère également de celui de Sextus. Le pyrrhonisme a donc connu une histoire marquée par différentes phases. C'est cette histoire qu'entend retracer Bett (principalement dans le chapitre 4). Plutôt que suspendre son jugement, étant donnée l'équipollence des arguments, du moins tel que le recommande Sextus dans le livre I des PH, Pyrrhon affirme, nous dit Bett, que puisque la réalité est indéterminée, nos opinions ne peuvent être ni vraies ni fausses.

L'étude de Bett est minutieusement construite, tout à fait dans le style de la tradition analytique anglo-saxonne. Il ne saurait en être autrement. Puisque la vérité historique nous échappera sans doute toujours. Les sources documentaires concernant Pyrrhon étant très réduites, les interprétations doivent être jaugées uniquement par leur cohérence avec d'autres textes secondaires. Dans le chapitre 1, intitulé «Pyrrho the Non-Sceptic», Bett analyse de manière méticuleuse le seul texte sur la pensée de Pyrrhon qui ne semble pas être contaminé par le pyrrhonisme tardif d'Énésidème et de Sextus. Bett y décèle la thèse métaphysique évoquée plus haut. Dans le chapitre 2, "Put it into Practice", l'auteur analyse les nombreuses anecdotes relatives à la vie extraordinaire du premier skeptikos, en examinant en particulier les fragments de son disciple, Timon de Phlionte (vers 325-235). Au chapitre 3, l'auteur cherche les précurseurs de Pyrrhon. Platon serait le philosophe qui aurait exercé une influence, directe ou indirecte, sur Pyrrhon. Enfin, dans le chapitre 4, Bett explique comment la pensée sceptique s'est transformée ultérieurement avec Énésidème et Sextus.

Étant donné l'intérêt que présente la thèse étonnante d'indéterminabilité qu'impute Bett à Pyrrhon, je vais, dans ce qui va suivre, présenter la démarche de l'auteur qui l'a conduit à cette thèse. Il me semble que Bett a mis le doigt sur un point important, cardinal, d'une portée considérable. Mais je ne saurais dire s'il a raison ou tort.

Le texte névralgique qui fait l'objet du litige opposant l'auteur à ses adversaires (entre autres, Jonathan Barnes, voir son compte rendu de l'étude de Bett dans Mind (vol. 110, octobre 2001), est le fameux passage d'Eusède de Césarée, évêque et théologien de l'Église d'Orient (né vers 260, mort en 339), où ce dernier cite, dans sa Préparation évangélique, un philosophe péripatéticien, Aristoclès de Messine, ayant vécu, lui, vers la fin du $1^{\text {er }}$ siècle, et qui, dans son ouvrage De la philosophie, aujourd'hui perdu, expose succinctement la philosophie de Pyrrhon. Péripatéticien, Aristoclès n'est pas l'ami des pyrrhoniens. Eusèbe non plus, loin de là. Le texte en question est donc un passage d'Aristoclès, cité par Eusèbe, lequel Aristoclès cite Timon présentant la pensée de Pyrrhon... Le lecteur comprend ici que notre connaissance la plus ancienne de Pyrrhon repose sur plusieurs "couches sédimentées». En somme, sur un rapport de troisième main. Bett est d'avis que le texte d'Aristoclès cité par Eusèbe est fiable, car il serait exempt de contamination ultérieure. De plus, Timon fut le proche disciple de Pyrrhon. Toute la question est de savoir si Aristoclès cite directement un ouvrage de Timon, le Python, selon toute vraisemblance, ouvrage aujourd'hui perdu, ou si Aristoclès reformule en ses propres mots la source apparemment pyrrhonienne qu'il a 
sous les yeux. En tout cas, d'après Bett, nous n'aurions aucune bonne raison de mettre en doute le compte rendu d'Aristoclès de la pensée de Pyrrhon. Personnellement, Bett ne m'a pas convaincu. Il est toujours possible qu'Aristoclès soit lui-même l'auteur du passage en question. En tout cas, aucun doute que le début du dit passage est de sa main. Comme ce texte est au cœur de l'interprétation de la pensée de Pyrrhon, je m’empresse de le citer dans son entièreté:

Il est avant tout nécessaire de mener une recherche sur notre propre connaissance. En effet, si nous sommes ainsi faits que nous ne connaissons rien, ce n'est pas la peine d'examiner le reste. Il s'en est trouvé parmi les anciens, eux aussi, qui l'ont déclaré, et Aristote a argumenté contre eux. Pyrrhon d'Élis lui aussi a défendu cette position avec force. Il n’a lui-même rien laissé par écrit, mais son élève Timon dit que quiconque veut atteindre le bonheur doit considérer ces trois questions : premièrement, que sont les choses par nature? deuxièmement, comment devons-nous être disposés à leur égard ? enfin, qu'en résultera-t-il pour ceux qui ont cette attitude? Selon lui, Pyrrhon déclarait que les choses sont également indifférentes, non évaluables, indécidables. Pour cette raison, nos sensations et nos opinions ne donnent ni la vérité ni la fausseté. Pour cette raison, il ne faut en rien leur faire confiance, mais il nous faut être sans opinion, sans inclinaison, inébranlables, en disant à propos de chaque chose en particulier pas plus qu'elle n'est ou qu'elle n'est pas, ou à la fois qu'elle est et n'est pas, ou que ni elle est ni elle n'est pas. Ce qui en résultera pour ceux qui se comportent effectivement ainsi, disait Timon, c'est d'abord l'usage parcimonieux de la parole, ensuite l'imperturbabilité, et, selon Énésidème, le plaisir. Voilà les points principaux de ce qu'ils disent.» (cité dans Long et Sedley, Les philosophes hellénistiques, tome 1, traduction par Jacques Brunschwig et Pierre Pellegrin, Paris, Garnier-Flammarion, 2001, p. 41, 1F.)

Comme la réponse aux deux autres questions dépend de la réponse à la première, celle-ci devient particulièrement névralgique : que sont les choses par nature? La question est épineuse, et la réponse, on s'en doute bien, fait l'objet d'une controverse. Quelle était donc la réponse de Pyrrhon? Le texte dit : Pyrrhon déclarait que les choses sont également indifférentes, non évaluables, indécidables. Qu'est-ce à dire? Il y a deux manières de comprendre cette réponse. Bett distingue l'interprétation épistémologique de l'interprétation métaphysique de la même réponse. D'après l'interprétation épistémologique, nous ne serions pas en mesure de connaître la nature des choses, c.-à-d. que nos capacités cognitives ne nous permettent pas de connaître ce qu'elles sont. C'est pourquoi les choses sont également indifférentes, non évaluables, indécidables. C'est d'ailleurs ce que laisse aussi entendre Aristoclès au tout début du texte. D'après lui, le pyrrhonien dénie aux humains la capacité de connaître. La réalité serait indéterminable en raison des limites inhérentes à nos capacités cognitives. C'est cette interprétation "épistémologisante» du scepticisme qui s'est imposée par la suite avec Sextus et que la tradition nous a léguée. Mais il n'est pas assuré que ce soit là la véritable interprétation à donner de la réponse de Pyrrhon à la question de savoir ce que sont les choses par nature. D'après Bett, en effet, la réponse de Pyrrhon n'est pas de nature épistémologique mais de nature métaphysique. D’après Bett, pour Pyrrhon, la raison pour laquelle «nos sensations et nos opinions ne sont ni vraies ni fausses ", c'est que les choses sont en elles-mêmes intrinsèquement «indifférentes, non évaluables, indécidables », c.-à-d. : les choses sont indéterminées. Pour éviter toute ambiguïté, Bett préfère le mot «indéterminé» à «indéterminable», ce dernier mot invitant à épouser la thèse épistémologique (p. 19). Pour Pyrrhon, donc, la réalité est 
par nature indéterminée. En somme, c'est parce que le monde est intrinsèquement ( «métaphysiquement») indéterminé, ou encore indéfini, que "nos sensations et nos opinions ne sont ni vraies ni fausses".

Laquelle de ces deux interprétations de la réponse de Pyrrhon est la vraie? D'après Bett, c'est l'interprétation métaphysique — la sienne — qui est vraie. Pourquoi ? Parce qu'elle s'harmonise davantage avec l'ensemble du passage d'Aristoclès. Au contraire, si l'on adopte l'interprétation épistémologique, le passage n'a plus de sens. En effet, dans ce dernier cas, si effectivement la nature des choses est indéterminable (en raison de nos capacités cognitives déficientes), alors on ne pourra pas dire que nos sensations et nos opinions sont ni vraies ni fausses, car pour pouvoir dire qu'elles sont telles, il faut être en mesure de connaître ce à quoi elles correspondent, ce qu'on a exclu au départ en présumant que la nature des choses est indéterminable. Si, au contraire, on adopte l'interprétation métaphysique, la réponse a du sens, car, la réalité étant indéterminée, cela seul explique que nos sensations et nos opinions ne soient ni vraies ni fausses. À mon avis, cet argument de Bett est puissant et décisif.

Pyrrhon serait donc "dogmatique». Il «dogmatise» en affirmant en effet quelque chose sur la nature intrinsèque de la réalité. Sextus, dans le premier livre des $\mathrm{PH}$, condamne cette attitude sans appel, car le sceptique ne peut pas se prononcer sur la nature de la réalité sous peine d'incohérence. Pyrrhon, lui, n'a pas ce scrupule. Pour lui, les propriétés des choses que nous observons - telles que être sucré, être rouge, être grand, être bien ou bon, etc., — sont foncièrement indéterminées ou indéfinies dès le départ. Et c'est précisément pour cette raison que Pyrrhon peut inférer que nos sensations et nos opinions ne donnent ni la vérité ni la fausseté.

De là découle également la réponse à la deuxième question du passage d'Aristoclès qui demande quelle attitude on devrait adopter devant le caractère indéterminé des choses : Il ne faut en rien faire confiance [à nos sensations ainsi qu'à nos opinions], mais il nous faut être sans opinion, sans inclinaison, inébranlables... En effet, puisque nos opinions ne sont ni vraies ni fausses, il faut éviter les malentendus en évitant d'avoir quelque opinion que ce soit, ou même d'avoir une inclinaison pour une opinion plutôt que pour une autre. Par ailleurs, il faut dire, continue le texte, à propos de chaque chose en particulier pas plus qu'elle n'est ou qu'elle n'est pas, ou à la fois qu'elle est et n'est pas, ou que ni elle est ni elle n'est pas. En somme, il faut dire à propos de chaque chose qu'elle n'est pas plus telle et telle que son contraire. On pourrait le croire, dès lors que, comme le recommande Sextus, Pyrrhon invoque ici la fameuse "suspension du jugement» (épochè). Nenni. D’abord, Pyrrhon n'utilise jamais l'expression technique épochè. Et quand Pyrrhon utilise l'expression 'pas plus' (ou mallon ou ouden mallon), celle-ci a une toute autre signification que celle que lui donne Sextus. (p. 30-31) En effet, si l'on admet la prémisse métaphysique suivant laquelle la réalité est indéterminée - ce que Pyrrhon admet, contrairement à Sextus — il n’y a dès lors plus de raison de «suspendre le jugement » quant à savoir, par exemple, si la tomate est rouge ou non. Car, pour Pyrrhon, la réponse à cette question est évidente : la tomate n'est pas rouge de manière déterminée, pas plus qu'elle n'est non rouge de manière déterminée; en somme : la tomate est tout autant rouge qu'elle n'est pas rouge.

Enfin, vient la réponse à la troisième question : qu'en résultera-t-il pour ceux qui ont cette attitude? La réponse découle en bonne partie, évidemment, des réponses précédentes. La traduction française (celle effectuée d'après la traduction anglaise de Long et Sedley) dit : ce qui en résultera pour ceux qui se comportent effectivement ainsi, disait Timon, c'est d'abord l'usage parcimonieux de la parole, ensuite l'imper- 
turbabilité... La traduction anglaise de Bett de ce même passage est littérale et, donc, plus adéquate: "Timon says that the result for those who are so disposed will be first speechless, but then freedom from worry..." (p. 16). Le mot speechless traduit mieux le grec aphasia, et freedom from worry : ataraxia. Une traduction française adéquate aurait été la suivante (nous reproduisons celle de Jacques Brunschwig, dans "Pyrrhon ", in Monique Canto-Sperber, dir., Philosophie grecque, Paris, PUF, 1997, p. 470) : "Pour ceux qui sont ainsi disposés, Timon dit que le bénéfice sera d'abord la perte de la parole (aphasia), puis l'exemption totale de trouble (ataraxia) [...]».

Donc, ayant admis la thèse d'indéterminabilité des choses qui, par suite, nous conduit à reconnaître que nos jugements sur les choses ne sont pas plus vrais que faux, il en résulte d'abord, cela va de soi, une sorte d'immense perplexité où nous restons pour ainsi dire bouche bée (aphasia); puis survient alors la tranquillité ou l'absence de trouble (ataraxia). Nous retrouvons ici les thèmes connus du silence, de l'ataraxie, qui ont fait pour ainsi dire la fortune du pyrrhonisme. Le pyrrhonisme, en fait, avant d'être considéré comme une théorie épistémologique, tel que le fera le scepticisme moderne, était une voie de la sagesse, comme le furent d'ailleurs toutes les philosophies anciennes. D'ailleurs, c'est la voie eudémoniste menant vers le bonheur. Comment est-il possible de vivre sans croyance? Pyrrhon, selon les témoignages anciens - ceux de Timon en particulier — était parvenu à cet égard à un haut degré de maîtrise de soi et d'imperturbabilité.

Cela étant, puisque le lecteur n'est pas, lui, parvenu au même degré d'imperturbabilité, nul doute qu'il est avide de connaître la réponse à une question cruciale restée en suspend jusqu'ici : qu'est-ce qui motive la thèse d'indéterminabilité ? Quel argument, en somme, avance Pyrrhon pour justifier sa thèse? La réponse de l'auteur se trouve dans le chapitre 3 , section 1 , The inderterminacy thesis : its nature and motivation. Voyons voir.

Quelle raison aurait avancé Pyrrhon pour croire que la réalité est foncièrement indéterminée? Il ne saurait s'agir ici que d'une hypothèse vraisemblable. D'après Bett, Pyrrhon serait parvenu à cette thèse en raison de la diversité (variability) conflictuelle d'après laquelle les choses se présentent à nous : le même objet en effet présente une diversité d'impressions à différentes personnes ou pour la même personne à différents moments, sous différentes conditions, "owing to changes in the objects themselves» (p. 114-115). Le raisonnement serait donc le suivant (cf. p. 117) :

1. Les choses se présentent elles-mêmes d'une multitude de façons conflictuelles entre elles;

2. Par conséquent, les choses n'ont pas de mode d'être qui soit stable;

3. La réalité est donc indéterminée.

Comme on l'a vu précédemment, de (3) Pyrrhon recommande de dire de chaque chose en particulier pas plus qu'elle n'est ou qu'elle n'est pas, ou à la fois qu'elle est et n'est pas, ou que ni elle est ni elle n'est pas. Considérons l'exemple suivant que discute l'auteur (p. 117). Sous un certain éclairage, à un certain moment de la journée, une pierre paraît brune; sous un autre éclairage, à un autre moment du jour, la même pierre semble orangée. Dans ces conditions, sommes-nous en droit de dire que la pierre est véritable brune ou orange? Non, répond Pyrrhon. Nous sommes en mesure de dire quelque chose concernant la couleur de la pierre, à savoir qu'elle est tantôt brune, tantôt orange; de plus, la pierre ne peut être, en elle-même, soit brune 
ou autre que brune. Conclusion : aucun des prédicats (brun ou orange) ne détermine la véritable nature de la pierre. On ne peut pas non plus dire que la pierre n'est pas en elle-même colorée, car, alors, on contreviendrait à la thèse d'indéterminabilité; en effet, dans ce cas, on se prononce de manière déterminée au sujet de la pierre, à savoir qu'elle n'a pas de couleur. "Pyrrho's thought, rather, would have to be that the rock is not in its true nature either brown or other than brown, but that nor is it, in its true nature, neither brown nor other than brown. " (ibid). Eu égard à la couleur, la pierre est donc indéterminée : "the predicates "brown" and "other than brown" neither apply to it nor fail to apply to it. »

On pourrait accuser de circularité le raisonnement de Pyrrhon - à supposer que ce soit bien le raisonnement qu'ait tenu Pyrrhon — car le fait que tout prédicat s'applique ni se s'applique est présenté comme une conséquence de la thèse d'indéterminabilité; celle-ci, par ailleurs, est présentée comme la conséquence du fait que tout prédicat s'applique et ne s'applique pas. À cela Bett réplique (note 10, p. 117-118) que la thèse d'indéterminabilité est en réalité précisément le fait que tout prédicat s'applique ni se s'applique à quoi que ce soit. Bett écrit : "The reasoning is therefore as follows: (1) things strike us in variable and conflicting ways; (2) any predicate that we might be inclined to apply to something in fact neither applies to it nor fails to apply to it - in other words, reality is indeterminate; so (3) we should speak in a way that reflects this indeterminacy, neither applying nor refusing to apply any particular predicate to any particular thing. " (ibid).

Je ne sais pas si la réplique de Bett l'absout de toute circularité. Personnellement, il me semble que non. Pour reprendre le joli mot de Jonathan Barnes (article cité plus haut), je ne suis pas plus certain que Bett ait raison qu'il ait tort. Chose certaine, en tout cas, l'histoire du scepticisme ne sera désormais plus la même. 